\title{
Implementation of a Monolithic Single Proof-Mass Tri-Axis Accelerometer Using CMOS-MEMS Technique
}

\author{
Chih-Ming Sun, Ming-Han Tsai, Yu-Chia Liu, and Weileun Fang, Member, IEEE
}

\begin{abstract}
This paper presents a novel single proof-mass tri-axis capacitive type complementary metal oxide semiconductormicroelectromechanical system accelerometer to reduce the footprint of the chip. A serpentine out-of-plane ( $Z$-axis) spring is designed to reduce cross-axis sensitivity. The tri-axis accelerometer has been successfully implemented using the TSMC 2P4M process and in-house postprocessing. The die size of this accelerometer chip containing the MEMS structure and sensing circuits is $1.78 \times 1.38 \mathrm{~mm}$, a reduction of nearly $50 \%$ in chip size. Within the measurement range of $0.8 \sim 6 \mathrm{G}$, the tri-axis accelerometer sensitivities (nonlinearity) of each direction are $0.53 \mathrm{mV} / \mathrm{G}(2.64 \%)$ for the X-axis, $0.28 \mathrm{mV} / \mathrm{G}(3.15 \%)$ for the Y-axis, and $0.2 \mathrm{mV} / \mathrm{G}(3.36 \%)$ for the Z-axis, respectively. In addition, the cross-axis sensitivities of these three axes range from $1 \%$ to $8.3 \%$ for the same measurement range. The noise floors in each direction are $120 \mathrm{mG} / \mathrm{rtHz}$ for the $\mathrm{X}$-axis, $271 \mathrm{mG} / \mathrm{rtHz}$ for the Y-axis, and $357 \mathrm{mG} / \mathrm{rtHz}$ for the $\mathrm{Z}$-axis.
\end{abstract}

Index Terms-Complementary metal oxide semiconductor (CMOS)-MEMS, tri-axis accelerometer.

\section{INTRODUCTION}

$\mathbf{S}$ MALL SIZE, low cost, multifunction, low power consumption, and easy integration with consumer electronics are some of the primary design issues for commercial microelectromechanical system (MEMS) applications such as inertia sensors and microphones. Various approaches have been reported to meet the related design requirements. The fabrication of micromechanical components using existing IC foundries provides a promising option to realize MEMS sensors. MEMS sensors can be fabricated by means of a complementary metal oxide semiconductor (CMOS) process together with postCMOS processing, named the CMOS-MEMS process [1]. This

Manuscript received December 15, 2009; revised March 30, 2010; accepted April 1, 2010. Date of publication May 20, 2010; date of current version June 23,2010. This project was supported in part by the NSC of Taiwan under Grants 96-2628-E-007-008-MY3 and 95-2221-E-007-068-MY3. The review of this paper was arranged by Editor A. M. Ionescu.

C.-M. Sun, M.-H. Tsai, and Y.-C. Liu are with the Institute of NEMS, National Tsing Hua University, Hsinchu 30013, Taiwan (e-mail: d9535812@oz.nthu.edu.tw; d9635816@oz.nthu.edu.tw; s9835804@m98.nthu. edu.tw).

W. Fang is with the Institute of NEMS and the Department of Power Mechanical Engineering, National Tsing Hua University, Hsinchu 30013, Taiwan (e-mail: fang@pme.nthu.edu.tw).

Color versions of one or more of the figures in this paper are available online at http://ieeexplore.iee.org.

Digital Object Identifier 10.1109/TED.2010.2048791 process allows the monolithic integration of IC and MEMS sensors to be achieved, and fulfills some of the aforementioned design requirements.

The integration of smaller MEMS inertial sensor has many applications such as in automobile industry, consumer electronics (e.g., digital cameras, mobile phones, notebooks, and video game systems), etc. Thus, the need for multiaxes (dual-axis or tri-axis) accelerometers is increasing. Various batch fabrication technologies, such as surface micromachining [2], bulk micromachining [3], SOI processes [4], and CMOS technology [5]-[9], have been reported to realize MEMS accelerometers. The tri-axis MEMS accelerometer has been extensively investigated in [3]-[7]. In [9], [10], the integration of tri-axis MEMS accelerometer was exploited for video game motion-controllers [11]. Multiproof-mass designs for in-plane and out-of-plane sensing are adopted in most reported monolithic multiaxis accelerometers [3]-[5]. Multiproof-mass accelerometers have the advantages of less crosstalk between sensing axes and relatively easier to design; however, a larger chip size is required.

It is possible to reduce the size, as well as the cost, by implementing a tri-axis MEMS accelerometer using a single proof-mass design. For instance, as reported in [5], [12], a reduction of nearly $50 \%$ in chip size can be achieved by a single proof-mass tri-axis accelerometer design. However, the complicated mechanical structures and sensing electrodes of the single proof-mass tri-axis accelerometer are critical design considerations. The materials and thin film layer stacking of standard CMOS process are fixed, and post-CMOS releasing processes are limited. It is even challenging to design a single proof-mass tri-axis accelerometer using existing CMOSMEMS processes, especially the out-of-plane (Z-axis) sensing component. The tri-axis accelerometers in [5], [6] establish a nonstandard CMOS process to implement parallel-plate gapclosing out-of-plane sensing electrodes. However, differential type capacitive sensing is not available. A differential verticalcomb out-of-plane sensing electrode for tri-axis accelerometer is reported in [7], [9]. This paper presents a novel single proof-mass tri-axis capacitive CMOS MEMS accelerometer to reduce the footprint of a chip. The tri-axis accelerometer consists of three differential capacitive sensing units for each axis. The approach in [14] is employed in this paper for the Zaxis sensing unit. A serpentine out-of-plane spring and sensing finger structures are designed to reduce the cross-axis sensitivity. The accelerometer has been implemented using TSMC $0.35 \mu \mathrm{m} 2 \mathrm{P} 4 \mathrm{M}$ CMOS process plus the present postrelease technique. 
(a)

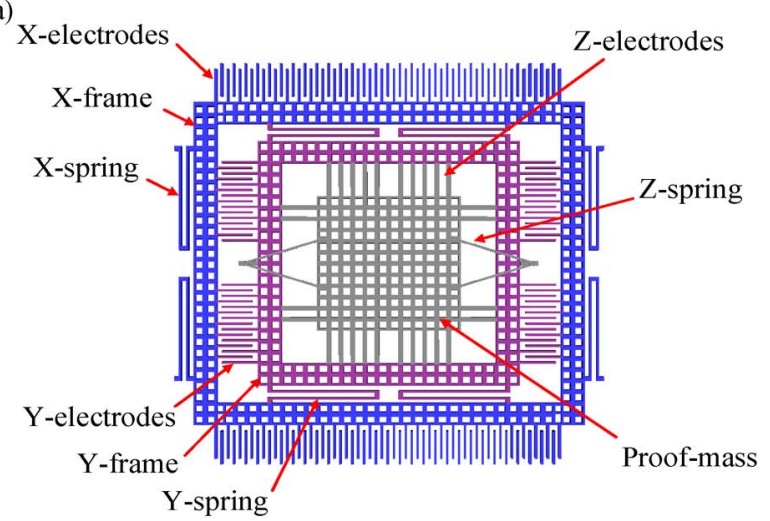

(b)

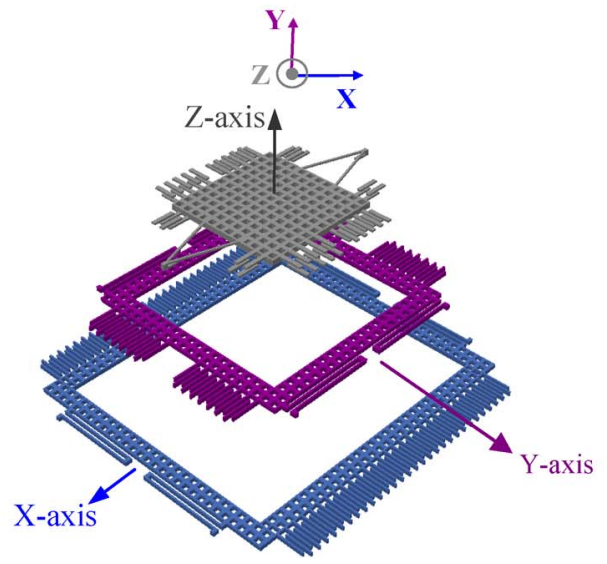

Fig. 1. Design concept of single proof-mass tri-axis accelerometer. (a) Top view, (b) exploded drawing of the sensing units for three different axes.

\section{DESIGN CONCEPT}

Fig. 1 shows the schematic design of the present tri-axis accelerometer. As shown in Fig. 1(a), the accelerometer consists of a proof-mass, two supporting frames (named $X-$ and Y-frame), and three sets of springs (named X-, Y-, and Z-spring) and sensing electrodes (named X-, Y-, and Z-electrodes). The accelerations in the three orthogonal axes are respectively detected by the capacitance change of the gap-closing combfinger electrodes. The three sets of springs are designed to be only flexible in one axis (the sensing axis) to suppress the unwanted motions of the sensing electrodes. Fig. 1(b) shows an exploded drawing of the accelerometer in Fig. 1(a). Each of the three primary sensing components shown in Fig. 1(b) consist of sensing electrodes and springs. These three components form the three orthogonal sensing units of the tri-axis accelerometer.

According to [13], the sensitivity of accelerometer can be expressed as

$$
\text { Sensitivity }=\frac{\Delta V_{\text {out }}}{A_{\mathrm{cc}}}=\underbrace{\frac{M}{K}}_{\text {Gain1 }} \times \underbrace{\frac{C_{0}}{g_{0}}}_{\text {Gain2 }} \times \underbrace{\frac{4 V_{m}}{\left(C_{p}+2 C_{0}\right)}}_{\text {Gain3 }} \times \underbrace{G_{\text {amp }}}_{\text {Gain4 }}
$$

where $A_{\mathrm{cc}}$ is the acceleration to be measured, $\Delta V_{\text {out }}$ is the output voltage, $M$ is the weight of the proof-mass, $K$ is the spring stiffness, $C_{0}$ is the net initial capacitance for all sensing electrode, $g_{0}$ is the initial gap between the stationary and moving comb-finger electrodes, $C_{p}$ is the parasitic capacitance, (a)

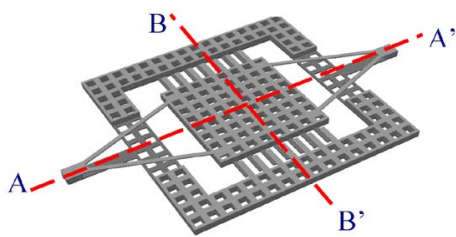

Z-axis sensing unit

(b)
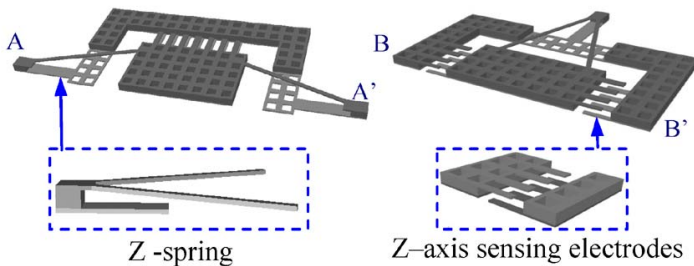

(c)
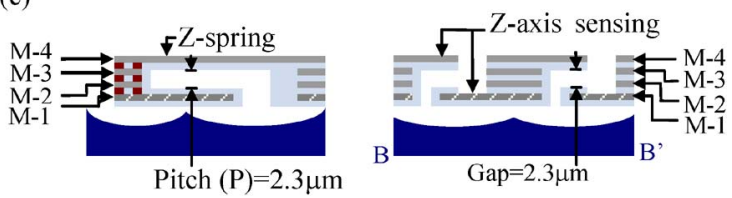

Fig. 2. Design concept of Z-axis sensing unit. (a) Bird's eye view, (b) crosssection view of Z-spring and Z-electrodes, and (c) the formation of the Z-spring and the Z-electrodes using the film stacking of CMOS layers.

$V_{m}$ is the input modulation voltage, and $G_{\text {amp }}$ is the circuit gain. Thus, Gain 1 refers to the multiplication of mechanical proof-mass $M$ and compliance $S(S=1 / K)$, Gain 2 refers to the net capacitance change $\Delta C$ of accelerometer resulting from the displacement of proof-mass $\Delta X$, as indicated by

$$
\left.\Delta C \cong \frac{\partial}{\partial g}\left(\frac{\varepsilon A}{g}\right)\right|_{g=g_{0}}=\frac{C_{0}}{g_{0}} \Delta x
$$

where $A$ is the sensing area, $\varepsilon$ is the dielectric constant. Gain3 can be yielded from

$$
\begin{aligned}
\Delta V & =V_{m}\left(\frac{C_{0}+\Delta C}{C_{p}+2 C_{0}}-\frac{C_{0}-\Delta C}{C_{p}+2 C_{0}}\right) \\
& -V_{m}\left(\frac{C_{0}-\Delta C}{C_{p}+2 C_{0}}-\frac{C_{0}+\Delta C}{C_{p}+2 C_{0}}\right)=\frac{4 V_{m}}{C_{p}+2 C_{0}} \Delta C
\end{aligned}
$$

where $\Delta V$ is the net voltage change from the readout circuit. The preamplifier in this paper acted as a buffer with a gain (Gain4) of one. The presented accelerometer sensitivity in the sensing axis can be predicted from (1).

\section{A. Out-of-Plane (Z-Axis) Sensing Unit}

The top component in Fig. 1(b) contains a proof-mass supported by the Z-spring to form the Z-axis sensing unit. As shown in Fig. 2(a), the Z-spring is also connected to the supporting frame. The illustrations in Fig. 2(b) show the cross-section views of Z-spring [AA'-section in Fig. 2(a)] and $\mathrm{Z}$-electrodes $\left[\mathrm{BB}^{\prime}\right.$-section in Fig. 2(a)]. The Z-spring is designed to be flexible only in the Z-axis (out-of-plane direction). As indicated in Fig. 2(b), the presented Z-spring is a serpentine structure folded in the out-of-plane direction. The Z-spring can thus span the Y-frame to increase its length and flexibility in the out-of-plane direction. Moreover, the V-shape of the Z-spring is used to increase the in-plane stiffness, allowing the in-plane 
TABLE I

Design Specifications of the Proposed Tri-Axis ACCElERometer

\begin{tabular}{|c|c|c|c|}
\hline & \multicolumn{3}{|c|}{ Spring Design } \\
\hline & $X$-spring & $Y$-spring & $Z$-spring \\
\hline $\begin{array}{l}\text { Spring Design } \\
\text { (top view) }\end{array}$ & $\frac{1}{6}$ & $\begin{array}{c}\rightarrow \\
0 \\
5 \\
(\mu \mathrm{m})\end{array}$ & $(\mu \mathrm{m})$ \\
\hline $\begin{array}{c}\text { Spring Design } \\
\text { (cross-section view) }\end{array}$ & 5 & $\left.\right|_{(\mu \mathrm{m})} ^{7} 7.06$ & $\underset{40}{\stackrel{2}{\longrightarrow}} \underset{(\mu \mathrm{m})}{\stackrel{2}{\longrightarrow}}$ \\
\hline Stiffness in X-axis $(\mathrm{N} / \mathrm{m})$ & 4.3 & 46.4 & 119.6 \\
\hline Stiffness in Y-axis $(\mathrm{N} / \mathrm{m})$ & 46.4 & 4.3 & 75.2 \\
\hline \multirow[t]{3}{*}{ Stiffness in Z-axis $(\mathrm{N} / \mathrm{m})$} & 256.8 & 256.8 & 3.9 \\
\hline & \multicolumn{3}{|c|}{ Sensing Electrode Design } \\
\hline & X-electrodes & Y-electrodes & Z-electrodes \\
\hline Sensing capacitance $(\mathrm{fF})$ & 39.6 & 28.5 & 15.3 \\
\hline $\begin{array}{l}\text { Capacitance change } \\
\text { sensitivity (fF/G) }\end{array}$ & 0.09 & 0.02 & 0.008 \\
\hline Length $(\mu \mathrm{m})$ & 40 & 40 & 42 \\
\hline Width $(\mu \mathrm{m})$ & 4.8 & 4.8 & 4 \\
\hline Thickness $(\mu \mathrm{m})$ & 7.06 & 7.06 & \\
\hline $\begin{array}{c}\text { Thickness (Top finger) } \\
(\mu \mathrm{m})\end{array}$ & & & 1.92 \\
\hline \multirow[t]{3}{*}{$\begin{array}{l}\text { Thickness (Bottom } \\
\text { finger) }(\mu \mathrm{m})\end{array}$} & & & 2.86 \\
\hline & \multicolumn{3}{|c|}{ Accelerometer Design Specifications } \\
\hline & $X$-axis Unit & Y-axis Unit & Z-axis Unit \\
\hline Sensitivity $(\mathrm{mV} / \mathrm{G})$ & 0.57 & 0.25 & 0.19 \\
\hline Natural frequency $(\mathrm{KHz})$ & 8.49 & 14.55 & 10.05 \\
\hline Noise Floors $(\mu \mathrm{G} / \mathrm{rtHz})$ & 137 & 253 & 305 \\
\hline $\begin{array}{c}\text { Cross-axis sensitivity } \_ \\
(\%)\end{array}$ & & 0 & 0 \\
\hline $\begin{array}{c}\text { Cross-axis sensitivity _Y } \\
(\%)\end{array}$ & 0 & & 0 \\
\hline $\begin{array}{c}\text { Cross-axis sensitivity } \_Z \\
(\%)\end{array}$ & 0 & 0 & \\
\hline
\end{tabular}

to out-of-plane stiffness ratio of Z-spring to be increased. In the TSMC 2P4M process, this paper selected the top metal layer (M4) and the bottom metal layer (M1) to form the folded beam. Fig. 2(c) shows the schematic illustration of films stacking for the proof-mass, spring, and sensing electrodes. The pitch $(\mathrm{P}=$ $2.3 \mu \mathrm{m})$ of the folded structure is realized by sacrificial metal etching. In addition, the top structure (M4) of folded Z-spring connects to the proof-mass, and the bottom structure (M1) of folded Z-spring connects to the Y-supporting frame. The joint of the top and bottom beams is formed by metal layers (M3 and M2) and tungsten vias. Table I summarizes the dimensions of a typical serpentine Z-spring design and its simulated stiffness. The in-plane to out-of-plane stiffness ratios for the Z-spring are 19 (Y-axis/Z-axis) and 31 (X-axis/Z-axis), respectively. In comparison, the in-plane to out-of-plane stiffness ratios of the existing design in [14] are only two (Y-axis/Z-axis) and three
(X-axis/Z-axis). In other words, after applying the in-plane (X-axis or Y-axis) acceleration, the displacement of the proposed Z-axis sensing unit is only $\sim 10 \%$ of that in [14]. Thus, the cross-axis sensitivity can be improved by the proposed Z-spring.

The planar dimensions of the Z-spring including the length, width, and angle of the folded beams can be easily changed and defined by the CMOS processes to a minimum line width of $0.6 \mu \mathrm{m}$. However, the thickness of the folded beam is limited to the layer stacks of the standard CMOS process. For instance, it is possible to select: 1) the top two metal layers (M4 + M3) and the bottom metal layer (M1); or 2) the top metal layers (M4) and the bottom two metal layer (M1 + M2), to form the folded Z-spring. However, in these two cases, the pitch between the folded Z-spring is defined by only one metal layer (M2 or $\mathrm{M} 3$ ), the pitch becomes $\mathrm{P}=\sim 0.6 \mu \mathrm{m}$ and could be too small for the folded Z-spring. To prevent the contact of top and bottom sensing electrodes, this paper employs two metal layers (M2 and M3) to define the sensing gap. The detailed process steps to realize this device will be discussed in the following section. The advantages of the folded Z-spring design are as follows: 1) the reduction of the out-of-plane spring constant and improvement of the in-plane stiffness; 2) the reduction of the footprint of the Z-spring area.

The Z-electrodes consist of a movable sensing electrode (on the proof-mass) and a stationary sensing electrode (on the supporting frame). As the accelerometer is applied with an out-ofplane acceleration, the proof-mass will move in the Z-axis, so as to cause the variation of the gap between sensing electrodes. The acceleration is detected by the capacitance change of the Z-electrodes. An increase of comb-finger length will increase the sensing capacitance of the Z-electrodes. However, as indicated in Fig. 2(c), the comb-finger electrode, consisting of various stacking layers, will be bent by the thin film residual stresses. The length of comb-finger needs to be considered to prevent the contact of the top and bottom sensing electrodes. Moreover, the metal layer is employed to act as the sacrificial layer to define the sensing gap. Since the sensing gap defined by one metal layer is less than $0.6 \mu \mathrm{m}$, this paper employs two metal layers (M2 and M3) to define the sensing gap to prevent the contact of the top and bottom sensing electrodes. In other words, the M4 metal layer is used as the top electrode, and the M1 metal layer acts as the bottom electrode.

Fig. 3 shows a schematic diagram illustrating the Z-axis fully differential capacitive sensing principle [14]. The movable $\mathrm{Z}$-axis sensing electrodes are attached to the proof-mass. As indicated in the $\mathrm{CC}^{\prime}$ cross section of Fig. 3(a), the left and right movable sensing electrodes are designed to be respectively located below and above the stationary sensing electrodes. On the other hand, at the $\mathrm{DD}^{\prime}$ cross-section, the left and right movable sensing electrodes are designed to be respectively located above and below the stationary sensing electrodes. After subjected to acceleration $(G)$ in the Z-axis, the proof-mass, as well as the movable sensing electrodes, will move in the outof-plane direction, leading to a sensing capacitance change. As indicated in Fig. 3(a), after the proof-mass moves downward, the capacitance changes of the sensing electrodes in $\mathrm{CC}^{\prime}$ are (left) $-\Delta \mathrm{C}$ and (right) $+\Delta \mathrm{C}$, and those in $\mathrm{DD}^{\prime}$ are (left) $+\Delta \mathrm{C}$ 
(a)
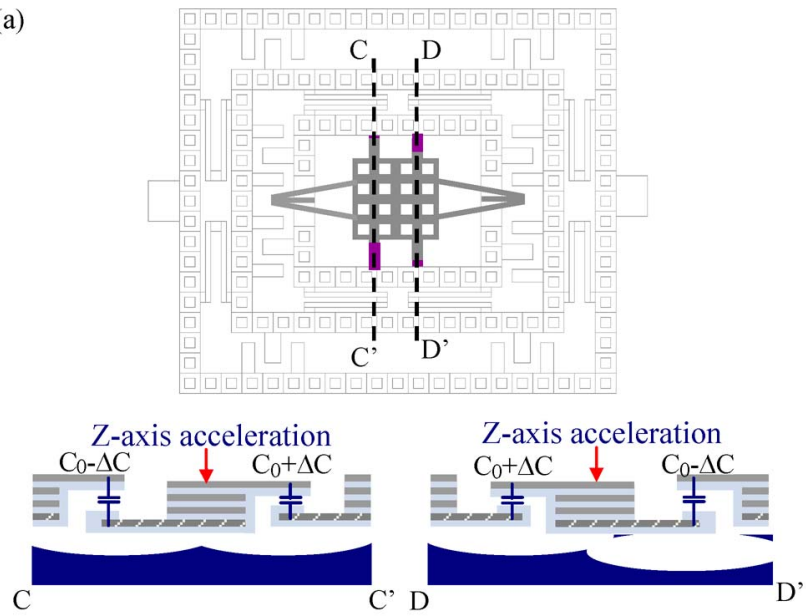

(b)

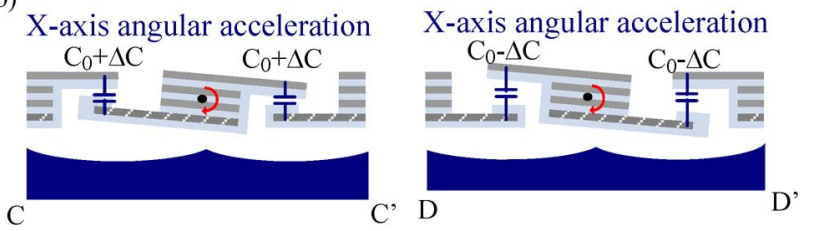

Fig. 3. Displacement and the related capacitance change of Z-axis sensing unit after excited by (a) the Z-axis linear acceleration and (b) the $\mathrm{X} / \mathrm{Y}$-axis torsional acceleration.

and (right) $-\Delta$ C. As a result, differential capacitive sensing is realized using the $\mathrm{Z}$-axis sensing electrode design, and the sensing voltage and sensitivity can be predicted from (1)-(3). As summarized in Table I, the typical designed initial capacitance in the $\mathrm{Z}$-axis is $15.3 \mathrm{fF}$ and its sensitivity along $\mathrm{Z}$-axis is $0.19 \mathrm{mV} / \mathrm{G}$. In addition, the proof-mass of the Z-axis sensing unit has an angular motion as the $\mathrm{X}$-axis angular acceleration applies on it. As indicated in Fig. 3(b), after the proof-mass rotate clockwise, the capacitance changes of the sensing electrodes in $\mathrm{CC}^{\prime}$ are (left) $+\Delta \mathrm{C}$ and (right) $+\Delta \mathrm{C}$, and those in $\mathrm{DD}^{\prime}$ are (left) $-\Delta \mathrm{C}$ and (right) $-\Delta \mathrm{C}$. After experiencing an $\mathrm{X}$-axis angular motion, the voltage change of the $\mathrm{Z}$-axis sensing unit can be expressed as

$$
\begin{aligned}
\left(\Delta V_{Z}\right)_{\theta X}=V_{m} & \left(\frac{C_{0}+\Delta C}{C_{p}+2 C_{0}}-\frac{C_{0}+\Delta C}{C_{p}+2 C_{0}}\right) \\
& -V_{m}\left(\frac{C_{0}-\Delta C}{C_{p}+2 C_{0}}-\frac{C_{0}-\Delta C}{C_{p}+2 C_{0}}\right)=0 .
\end{aligned}
$$

Thus, the net voltage change resulted from the angular motion of the Z-axis sensing unit can ideally be canceled by the fully differential sensing electrodes in this paper. Similarly, the crossaxis errors of the $\mathrm{Z}$-axis sensing unit from the $\mathrm{Y}$ - and $\mathrm{Z}$-axis torsional accelerations can also be canceled. Table II summarizes the typical simulated torsional stiffnesses of the Z-axis sensing unit. The predicted angular displacements and the voltage changes resulting from torsional accelerations are also indicated in Table II.

Moreover, the $\mathrm{Z}$-axis sensing unit has an in-plane displacement as the lateral acceleration (in $\mathrm{X}$ - or Y-axis) applying on it. As shown in Fig. 4(a) and (b), after the Z-axis experiences an $\mathrm{X}$-axis acceleration, the capacitances in both of the upperside (e.g., $\mathrm{Z}_{1}$ and $\mathrm{Z}_{3}$ ) and lowerside (e.g., $\mathrm{Z}_{2}$ and $\mathrm{Z}_{4}$ ) electrodes are
TABLE II

Summary of the Simulated Cross-AXis Sensitivity CaUSEd BY THE TORSIONAL ACCELERATION

\begin{tabular}{|l|c|c|c|}
\hline & \multicolumn{3}{|c|}{$\begin{array}{c}\text { Simulated Cross-Axis Sensitivity by } \\
\text { Torsional Acceleration }\end{array}$} \\
\hline & X-axis unit & Y-axis unit & Z-axis unit \\
\hline $\begin{array}{c}\text { Torsional spring constant in } \\
\mathrm{X} \text {-axis }(\mathrm{Nm} / \theta)\end{array}$ & 38.16 & 72.73 & 13.8 \\
\hline $\begin{array}{c}\text { Torsional spring constant in } \\
\text { Y-axis }(\mathrm{Nm} / \theta)\end{array}$ & 72.73 & 38.16 & 58.25 \\
\hline $\begin{array}{c}\text { Torsional spring constant in } \\
\mathrm{Z} \text {-axis }(\mathrm{Nm} / \theta)\end{array}$ & 56.54 & 56.54 & 45.6 \\
\hline $\begin{array}{l}\text { Angular motion due to } \mathrm{X} \text {-axis } \\
\text { angular acceleration }\left(\theta / \% / \mathrm{S}^{2}\right)\end{array}$ & $1.97 \times 10^{-5}$ & $6.87 \times 10^{-6}$ & $1.81 \times 10^{-5}$ \\
\hline $\begin{array}{l}\text { Angular motion due to } \mathrm{Y} \text {-axis } \\
\text { angular acceleration }\left(\theta / \% / \mathrm{S}^{2}\right)\end{array}$ & $1.03 \times 10^{-5}$ & $1.31 \times 10^{-5}$ & $4.29 \times 10^{-6}$ \\
\hline $\begin{array}{l}\text { Angular motion due to } \mathrm{Z} \text {-axis } \\
\text { angular acceleration }\left(\theta / \% / \mathrm{S}^{2}\right)\end{array}$ & $1.33 \times 10^{-5}$ & $8.84 \times 10^{-6}$ & $5.48 \times 10^{-6}$ \\
\hline $\begin{array}{l}\text { Voltage change due to } \mathrm{X} \text {-axis } \\
\text { angular acceleration }\left(\mathrm{fF} / \% / \mathrm{S}^{2}\right)\end{array}$ & 0 & 0 & 0 \\
\hline $\begin{array}{l}\text { Voltage change due to } \mathrm{Y} \text {-axis } \\
\text { angular acceleration }\left(\mathrm{fF} / \% / \mathrm{S}^{2}\right)\end{array}$ & 0 & 0 & 0 \\
\hline $\begin{array}{l}\text { Voltage change due to } \mathrm{Z} \text {-axis } \\
\text { angular acceleration }\left(\mathrm{fF} / \% / \mathrm{S}^{2}\right)\end{array}$ & 0 & 0 & 0 \\
\hline
\end{tabular}

decreased because of the decreasing overlapped area. According to the fully differential sensing electrodes design, the net capacitance change of the Z-axis sensing unit introduced by the $\mathrm{X}$-axis acceleration is

$$
\begin{aligned}
\left(\Delta V_{Z}\right)_{\mathrm{AX}}=V_{m} & \left(\frac{C_{0}-\Delta C}{C_{p}+2 C_{0}}-\frac{C_{0}-\Delta C}{C_{p}+2 C_{0}}\right) \\
& -V_{m}\left(\frac{C_{0}-\Delta C}{C_{p}+2 C_{0}}-\frac{C_{0}-\Delta C}{C_{p}+2 C_{0}}\right)=0 .
\end{aligned}
$$

Again, as shown in Fig. 4(c), the net capacitance change of the $\mathrm{Z}$-axis sensing unit introduced by the Y-axis acceleration is

$$
\begin{aligned}
\left(\Delta V_{Z}\right)_{\mathrm{AY}}=V_{m} & \left(\frac{C_{0}+\Delta C}{C_{p}+2 C_{0}}-\frac{C_{0}-\Delta C}{C_{p}+2 C_{0}}\right) \\
& -V_{m}\left(\frac{C_{0}+\Delta C}{C_{p}+2 C_{0}}-\frac{C_{0}-\Delta C}{C_{p}+2 C_{0}}\right)=0 .
\end{aligned}
$$

According to (5) and (6), the cross-axis sensitivity of the $\mathrm{Z}$-axis sensing unit resulting from the $\mathrm{X}$ - and $\mathrm{Y}$-axis accelerations, respectively, are canceled by the fully differential sensing electrodes design, as summarized in Table I.

\section{B. In-Plane (X-Axis and Y-Axis) Sensing Units}

As indicated in Fig. 5(a), the Z-axis-sensing unit connects to the Y-frame supported by the Y-spring to form the Y-axissensing unit. The Y-spring is designed to be flexible only in the Y-axis, as shown in Table I. The Y-electrodes also consist of a movable sensing electrode (on the Y-frame) and a stationary sensing electrode (on the $\mathrm{X}$-frame). As Y-acceleration is applied, the proof-mass and the Y-frame will move along the Y-axis, so the gap between Y-electrodes is changed. Thus, the Y-axis acceleration can be detected by the capacitance change of the Y-electrodes. For the in-plane sensing electrodes design, 
(a)

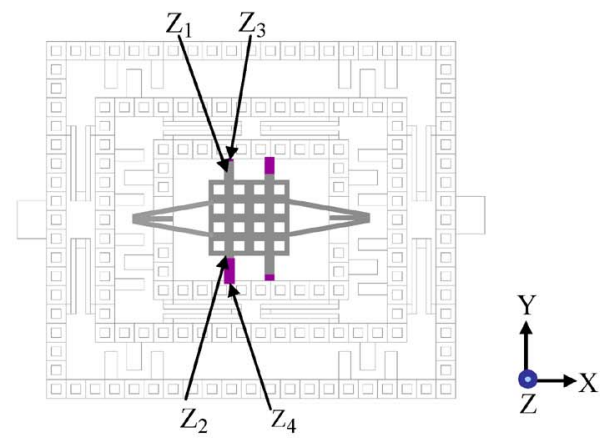

(b)

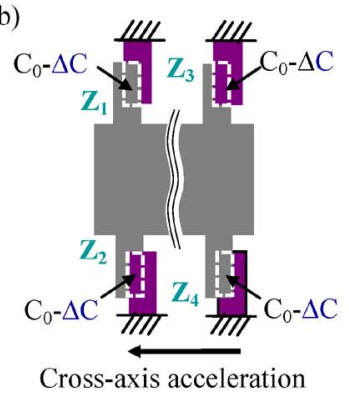

(c)

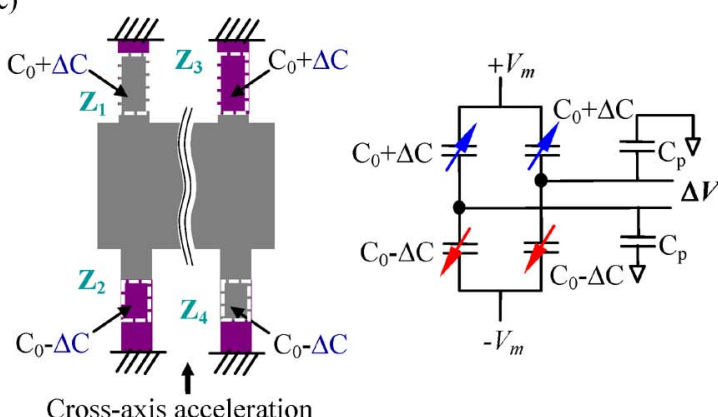

Fig. 4. Overlap area of sensing electrodes (in dashed line) for the combfingers of Z-axis sensing unit. (a) The case without in-plane motion, (b) the case with area change (capacitance change as well) of sensing electrodes induced by the $\mathrm{X}$-axis in-plane motion, and (c) the case with area change (capacitance change as well) of sensing electrodes caused by the Y-axis in-plane motion.

(a)

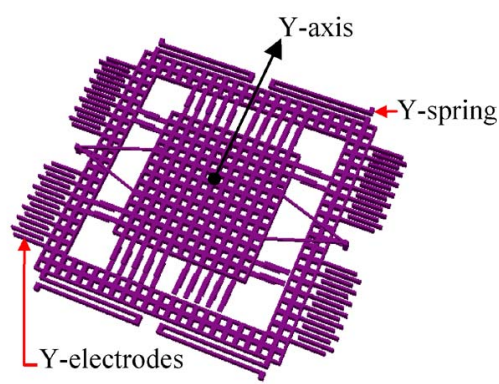

(b)

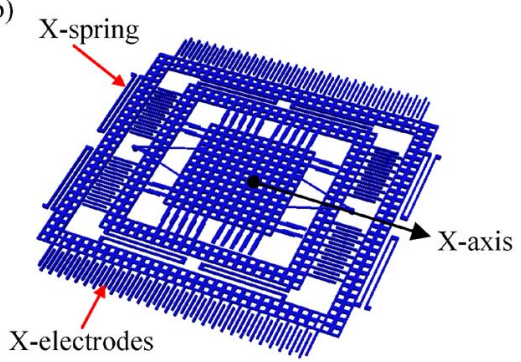

Fig. 5. Design concept of in-plane acceleration sensing units. (a) Y-axissensing unit, and (b) X-axis-sensing unit. (a)

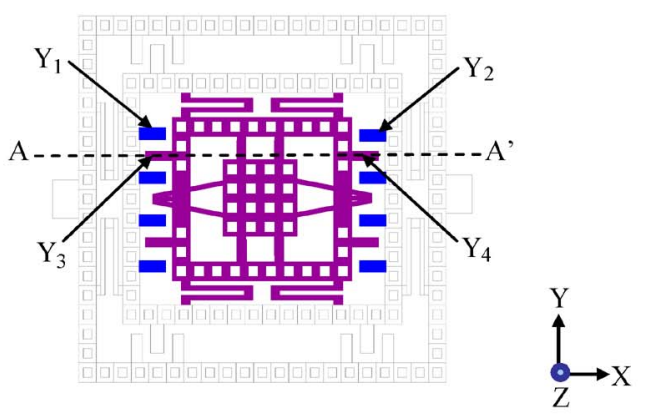

(b)

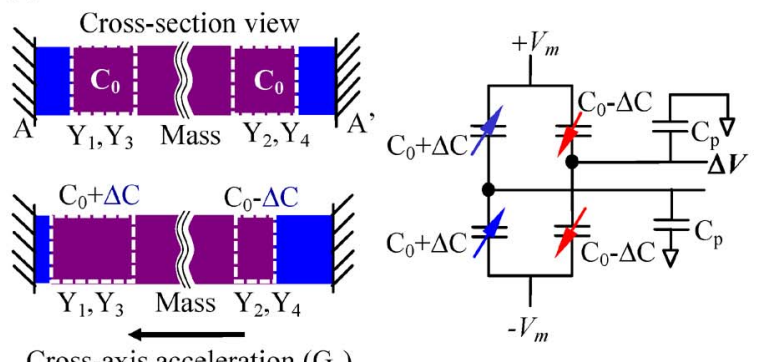

(c)

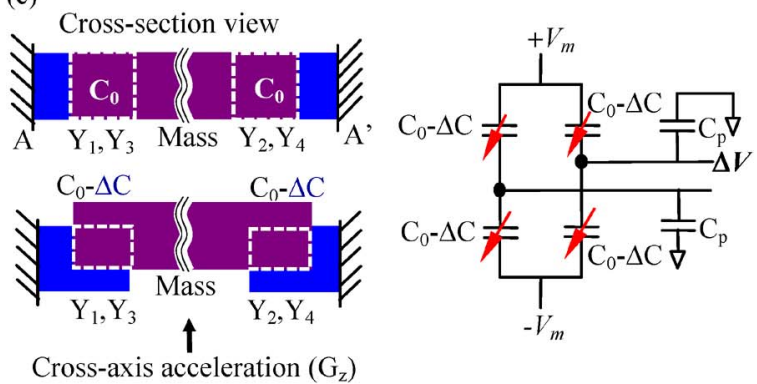

Fig. 6. Overlap area of sensing electrodes (in dashed line) for the combfingers of Y-axis-sensing unit. (a) Top-view of the accelerometer with Y-axis sensing unit highlighted, (b) the area change of sensing electrodes induced by the $\mathrm{X}$-axis in-plane motion, and (c) the area change of sensing electrodes caused by the Z-axis out-of-plane motion.

the comb-fingers should be as thick as possible to increase their overlap area and further increase the sensing capacitance. Thus, this paper employs all of the four metal layers and tungsten vias to form the comb-fingers. In this case, the equivalent proof-mass of the Y-axis-sensing unit (including the proofmass and the Y-frame) is larger than that of the Z-axis-sensing unit (only the proof-mass). Similarly, the Y-axis-sensing unit connects to the $\mathrm{X}$-frame supported by the $\mathrm{X}$-spring to form the $\mathrm{X}$-axis-sensing unit, as shown in Fig. 5(b). The X-axis- and Y-axis-sensing units have the same design concept and working principle. The designed initial capacitance (sensitivity) in the $\mathrm{X}$ - and Y-axis are, respectively, $39.6 \mathrm{fF}(0.57 \mathrm{mV} / \mathrm{G})$, and $28.5 \mathrm{fF}(0.25 \mathrm{mV} / \mathrm{G})$. The analyzed cross-axis sensitivity in $\mathrm{X}$ - and Y-direction are shown in Table I.

Fig. 6 shows the cross-axis sensitivity of the Y-axis-sensing unit. The $\mathrm{X}$-axis acceleration will cause the Y-axis-sensing unit to displace in the X-direction. Such displacement will lead to an overlap area variation in the sensing electrodes and will cause the capacitance of the sensing electrodes to change further. As indicated in Fig. 6(a) and (b), the capacitance in the lefthand-side electrodes $\left(\mathrm{Y}_{1}\right.$ and $\left.\mathrm{Y}_{3}\right)$ increases because of the increasing overlapped area. However, the capacitance of the 
right-hand-side electrodes $\left(\mathrm{Y}_{2}\right.$ and $\left.\mathrm{Y}_{4}\right)$ is decreased due to the decreasing overlapped area. As a result, according to the fully differential sensing electrodes design, the net capacitance change of the Y-axis-sensing unit introduced by the $\mathrm{X}$-axis acceleration is, according to (7), the net voltage change of the Y-axis-sensing unit resulted from the $\mathrm{X}$-axis acceleration can be canceled by the fully differential sensing electrodes

$$
\begin{aligned}
\left(\Delta V_{Y}\right)_{\mathrm{AX}}=V_{m}( & \left.\frac{C_{0}+\Delta C}{C_{p}+2 C_{0}}-\frac{C_{0}+\Delta C}{C_{p}+2 C_{0}}\right) \\
& -V_{m}\left(\frac{C_{0}-\Delta C}{C_{p}+2 C_{0}}-\frac{C_{0}-\Delta C}{C_{p}+2 C_{0}}\right)=0
\end{aligned}
$$

design. Similarly, the net capacitance change of the X-axissensing unit introduced by the $\mathrm{Y}$-axis acceleration is also canceled in the same manner. Moreover, the out-of-plane acceleration acting on the tri-axis accelerometer will also cause an out-of-plane linear displacement for both of the X-axisand Y-axis- sensing units. Thus, as shown in Fig. 6(c), the capacitances for both left-hand- and right-hand-sides sensing electrodes decrease because of the decreasing overlapped area. Again, according to the fully differential sensing electrodes design, the net voltage change of the X-axis- (or Y-axis-) sensing unit introduced by the Z-axis acceleration is

$$
\begin{aligned}
\left(\Delta V_{X}\right)_{\mathrm{AZ}}=V_{m} & \left(\frac{C_{0}-\Delta C}{C_{p}+2 C_{0}}-\frac{C_{0}-\Delta C}{C_{p}+2 C_{0}}\right) \\
& -V_{m}\left(\frac{C_{0}-\Delta C}{C_{p}+2 C_{0}}-\frac{C_{0}-\Delta C}{C_{p}+2 C_{0}}\right)=0 .
\end{aligned}
$$

In short, according to (7) and (8), the net voltage change resulted from the cross-axis interference for the $\mathrm{X}$-axis- and Y-axis-sensing units can be canceled by the fully differential sensing electrodes. The design results are also summarized in Table I. Moreover, as discussed in Section II-A, the net voltage change resulted from the angular motion of X-axis- and Y-axissensing units can also be canceled by the fully differential sensing electrodes in this paper. Table II summarizes the typical simulated torsional stiffnesses of the X-axis- and Y-axissensing units listed in Table I. After applying the angular accelerations to the accelerometer, the predicted angular displacements of the X-axis- and Y-axis-sensing units and the related voltage changes are also indicated in Table II.

The primary noise sources of the proposed accelerometer are Brownian noise and the input referred noise of the interface circuitry. The designed input referred noise, contributed by flicker noise and thermal noise are, respectively, $58 \mu \mathrm{G} / \mathrm{rtHz}$ (X-axis), $132 \mu \mathrm{G} / \mathrm{rtHz}$ (Y-axis), and $174 \mu \mathrm{G} / \mathrm{rtHz}$ (Z-axis), as estimated from the accelerometer design. Since the Brownian noise is mainly dominated by the proof-mass of the accelerometer, the total thickness of CMOS stacking layers (near $7 \mu \mathrm{m}$ ) gives limits the noise floor of the proposed design. Moreover, according to the proposed design, the Z-axis-sensing unit has the smallest proof-mass leading to the largest Brownian noise. The designed Brownian noises for the different sensing units are $124 \mu \mathrm{G} / \mathrm{rtHz}$ (X-axis), $216 \mu \mathrm{G} / \mathrm{rtHz}$ (Y-axis) and $250 \mu \mathrm{G} / \mathrm{rtHz}$ (Z-axis), respectively. As a result, the designed noise floors of the proposed accelerometer for the different sensing units are
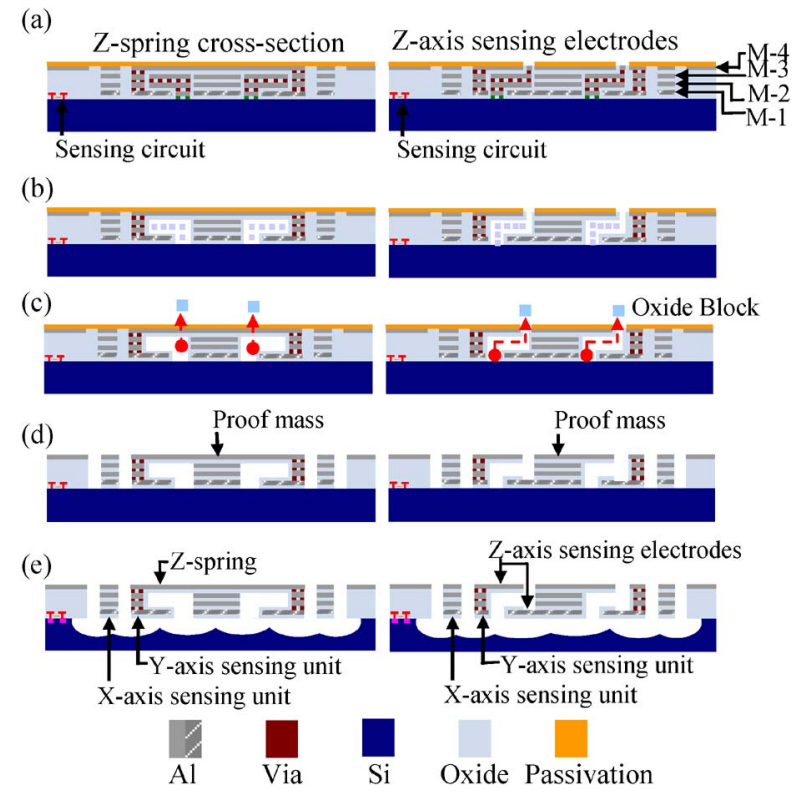

Fig. 7. Fabrication steps of the post-CMOS processes.

$137 \mu \mathrm{G} / \mathrm{rtHz}$ (X-axis), $253 \mu \mathrm{G} / \mathrm{rtHz}$ (Y-axis) and $305 \mu \mathrm{G} / \mathrm{rtHz}$ (Z-axis), respectively.

\section{CMOS-MEMS POSTPROCESSING}

Fig. 7 shows the fabrication process steps to realize the monolithic integrated single proof-mass tri-axis CMOS accelerometer. The two groups of cross-section views in Fig. 7 respectively indicate the process steps for the Z-spring $\left(\mathrm{AA}^{\prime}\right.$ cross section in Fig. 2) and out-of-plane sensing comb-fingers (BB' cross section in Fig. 2). The fabrication begins with the substrate prepared by standard TSMC $0.35 \mu \mathrm{m}$ CMOS $2 \mathrm{P} 4 \mathrm{M}$ processing. As shown in Fig. 7(a), this standard process offers one passivation layer, four aluminum metal layers (named M1 to M4), four silicon dioxide dielectric films, and several tungsten vias stacked and patterned on top of the silicon substrate. These stacked layers with a total thickness of nearly $7 \mu \mathrm{m}$ were then exploited to form the suspended micromachined structures. The post-CMOS processes consisted of wet etching of the metal layers, dry etching of dielectric layers, and isotropic bulk silicon etching from the frontside of substrate are illustrated in Fig. 7(b)-(e).

As shown in Fig. 7(a), the pattern and stacking designs of metal films and tungsten vias are clearly observed. These metal films were exploited as sacrificial layers and sensing electrodes. The passivation layer was patterned to define the region for following wet etching. As shown in Fig. 7(b), the solution containing $\mathrm{H}_{2} \mathrm{SO}_{4}$ and $\mathrm{H}_{2} \mathrm{O}_{2}$ was used to etch through the metal layers and tungsten vias [14], [15]. The passivation layer acted as a protection film during the metal wet etching. In addition, the dielectric film distributed along the path of the metal wet etching was also employed as a protection layer in this process. As the surrounding metal layers are fully removed, the dielectric blocks were released from the substrate, as illustrated in Fig. 7(b). After that, the dielectric blocks were removed away from the substrate, as indicated in Fig. 7(c). The thickness and the pitch of out-of-plane springs were determined, as shown in 


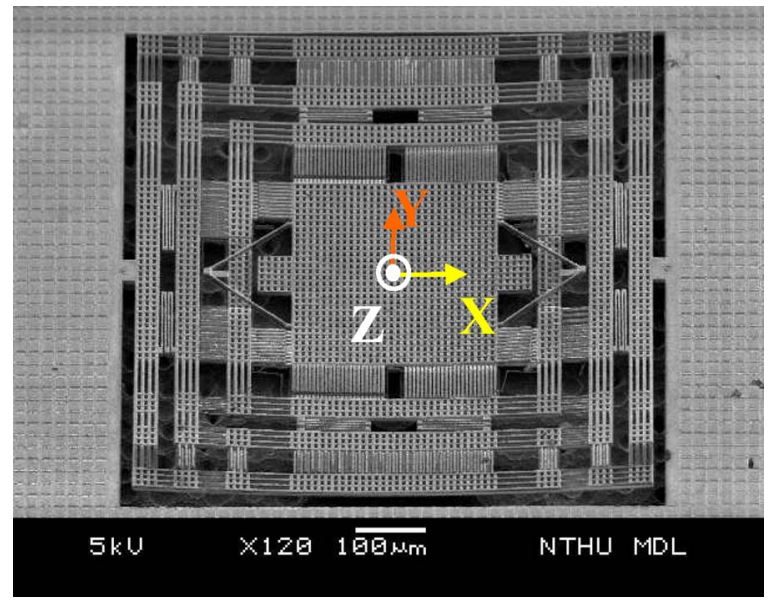

Fig. 8. SEM micrograph of a typical fabricated single proof-mass tri-axis accelerometer.

the left of Fig. 7(c). In addition, the gap between the top and bottom electrodes was also defined, as shown in the right of Fig. 7(c). After removing the top passivation layer, as illustrated in Fig. 7(d), RIE was employed to etch dielectric $\mathrm{SiO}_{2}$ layers (top metal layer M4 acted as etching mask) to define: 1) the shape of accelerometer; and 2) the gap between the stationary and the movable comb-fingers of in-plane accelerometers. Finally, dry isotropic bulk silicon etching using $\mathrm{XeF}_{2}$ was used to release the accelerometers, as shown in Fig. 7(e).

\section{RESUlts}

This paper has successfully demonstrated the tri-axis accelerometer using the process in Fig. 7. The scanning electron microscope (SEM) micrograph in Fig. 8 is a typical fabricated CMOS-MEMS single proof-mass tri-axis accelerometer $(770 \times 770 \mu \mathrm{m})$. As compared with the accelerometer in [16], this device reduces the chip footprint by nearly $50 \%$. Fig. 9(a) shows the key components of the tri-axis accelerometer, including the proof-mass, springs and sensing electrodes of different axes, and supporting frames. The silicon substrate underneath can also be observed. The rough surface of the silicon substrate is resulted from the $\mathrm{XeF}_{2}$ isotropic etching. The out-of-plane serpentine Z-spring is clearly observed in the zoom-in micrographs in Fig. 9(b). The top and bottom layers of the Z-spring are, respectively, fixed to the proof-mass and the Y-frame. In addition, as indicated in Fig. 9(b), the movable electrode (fixed to the proof-mass) is on top of the stationary electrode (fixed to the Y-frame) for one of the Z-electrodes. On the other hand, the stationary electrode is on top of the movable electrode for the other Z-electrodes. Thus, fully differential sensing electrodes in the out-of-plane direction are realized.

The CMOS circuits are monolithically integrated in this chip to form a standard two-stage cascode differential amplifier [17]. The preamplifier circuit acts as a buffer for output signal. The conversion gain is one to reduce the circuit noise. In this paper, there were three sets of CMOS circuits for each sensing axis. Fig. 10(a) further shows the accelerometer chip after wire bonding and packaging. The die size of this chip is $1.78 \times 1.38 \mathrm{~mm}$. To calibrate the performance of the presented accelerometer, a commercial tri-axis accelerometer

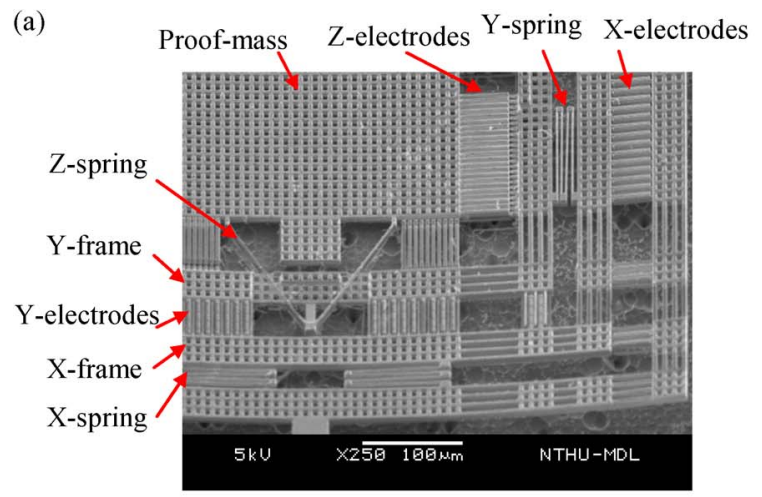

(b)

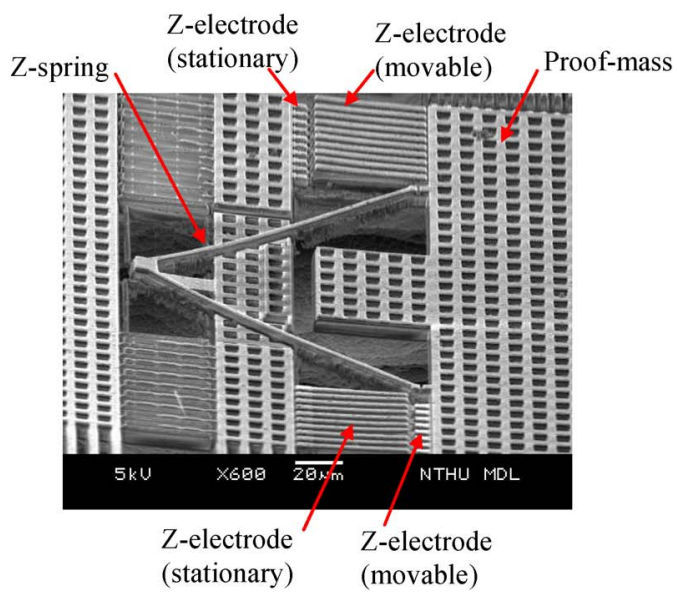

Fig. 9. Zoom-in SEM micrographs to show (a) the proof-mass, the frames, springs, and sensing electrodes, and (b) the serpentine Z-spring and the fully differential Z-electrodes. (a)

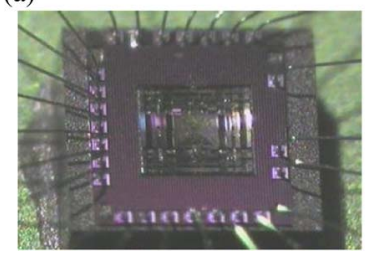

(b)

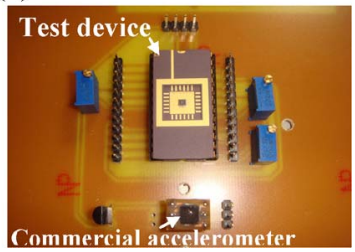

Fig. 10. Accelerometer chip, (a) after wire bonding and packaged in ceramic housing, and (b) assemble on the test board.

was also packaged on the test board, as shown in Fig. 10(b). The test setup in Fig. 11(a) was established to characterize the performance of the accelerometer. The test board shown in Fig. 10(b) was mounted to a position stage on the shaker. After that, the accelerometer was excited by the shaker with a given vibration amplitude and frequency. The dynamic characteristic of the shaker was monitored using the commercial accelerometer on the test board. The sensing signal was then measured and displayed using a spectrum analyzer. During the test, a modulation signal of $1 \mathrm{~V}$ at $1 \mathrm{MHz}$ was applied to the sensing chip. In this test, the excitation range provided by the typical sensitivities of the accelerometer are $0.53 \mathrm{mV} / \mathrm{G}$ in $\mathrm{X}$-axis, $0.28 \mathrm{mV} / \mathrm{G}$ in Y-axis, and $0.2 \mathrm{mV} / \mathrm{G}$ in Z-axis. This paper also employed a least squares regression to perform the linear fit of the measurement results in Fig. 11(b), and the nonlinearity can be expressed as [18]

$$
\text { Non-linearity }=\frac{\Delta V_{\max }}{\Delta V_{\text {full-span }}} \times 100 \%
$$


(a)

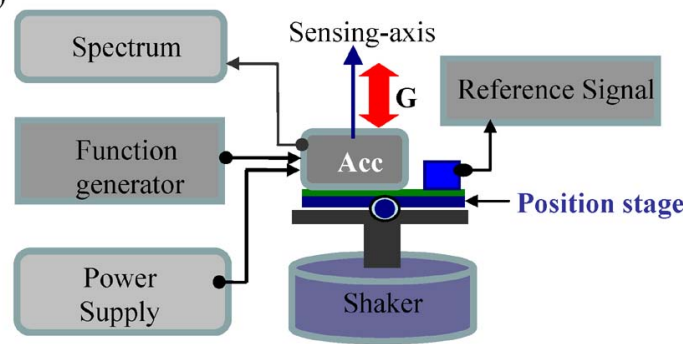

(b)

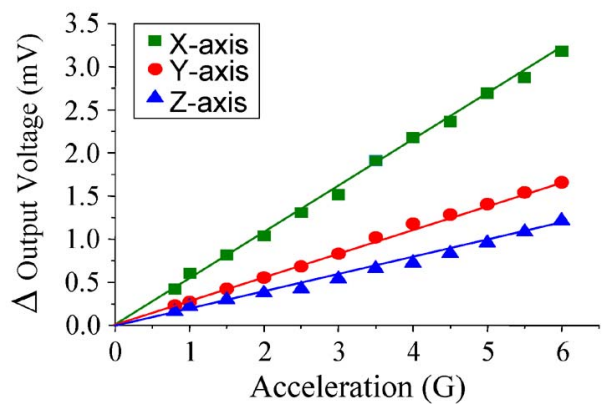

Fig. 11. (a) The test setup to characterize the performances of the tri-axis accelerometer, and (b) the typical measured sensitivities in three different axes.

TABLE III

Summary of the Measured Performances of a TYPICAL FABRICATED TRI-AXIS ACCELEROMETER

\begin{tabular}{|c|c|c|c|}
\hline & \multicolumn{3}{|c|}{ Measured Accelerometer Performances } \\
\hline & X-axis Unit & Y-axis Unit & Z-axis Unit \\
\hline Sensitivity (mV/G) & 0.53 & 0.28 & 0.20 \\
\hline Non-linearity (\%) & 2.64 & 3.15 & 3.36 \\
\hline Resonant Freq. (KHz) & $7.92 \pm 0.51$ & $15.75 \pm 0.39$ & $9.54 \pm 0.11$ \\
\hline Power consumption (mW) & \multicolumn{3}{|c|}{5} \\
\hline Noise floor (mG/rtHz) & 120 & 271 & 357 \\
\hline Cross-axis sensitivity_X(\%) & $/$ & $<7.46$ & $<2.88$ \\
\hline Cross-axis sensitivity_Y(\%) & $<1$ & $/$ & $<8.05$ \\
\hline Cross-axis sensitivity_Z (\%) & $<1$ & $<8.33$ & $/$ \\
\hline
\end{tabular}

shaker was from $0.8 \mathrm{G}$ to $6 \mathrm{G}$, and the excitation frequency was at $150 \mathrm{~Hz}$. The measurement results in Fig. 11(b) indicate that the where the $\Delta \mathrm{V}_{\max }$ is the maximum deviation between the measured and linear-fit voltages, and the $\Delta V_{\text {full-span }}$ is the full span voltage for the measurement. Moreover, the cross-axis signal coupling of the accelerometer was also characterized. As a result, the cross-axis sensitivity and the nonlinearity of accelerometer are less than $8.3 \%$ and $4 \%$, respectively, when sensing range between $0.8 \mathrm{G} \sim 6 \mathrm{G}$. Table III summarizes the characteristics of the presented accelerometer. The measured noise floors of each direction are $120 \mathrm{mG} / \mathrm{rtHz}$ of X-axis, $271 \mathrm{mG} / \mathrm{rtHz}$ of Y-axis, and $357 \mathrm{mG} / \mathrm{rtHz}$ of Z-axis, respectively. The noise is high because of the low sensitivity. The output noise in terms of voltage is about $75 \mu \mathrm{V} / \mathrm{rtHz}$. As indicated in Table III, the measured resonant frequencies (averaged from five measurements), along the $\mathrm{X}-$, Y-, and Z-axis are $7.92 \pm$ $0.51 \mathrm{kHz}, 15.75 \pm 0.39 \mathrm{kHz}$, and $9.54 \pm 0.11 \mathrm{kHz}$, respectively. According to the simulation, the ideal designed resonant frequencies (not considering the deviations caused by the mismatch of structural dimensions and material properties) along
TABLE IV

Comparison of The Presented AND the Existing Single PROOF-MASS TRI-AXIS MEMS ACCELEROMETERS

\begin{tabular}{|c|c|c|c|c|c|c|c|c|}
\hline & \multicolumn{2}{|c|}{ H. Takao [6] } & \multicolumn{2}{|c|}{ H. Qu [7] } & \multicolumn{2}{|c|}{ K. Kwon [19] } & \multicolumn{2}{|c|}{ This study } \\
\hline Process & \multicolumn{2}{|c|}{$\begin{array}{l}\text { SOI CMOS } \\
\text { MEMS }\end{array}$} & \multicolumn{2}{|c|}{$\begin{array}{l}\text { CMOS } \\
\text { MEMS }\end{array}$} & \multicolumn{2}{|c|}{ SOI MEMS } & \multicolumn{2}{|c|}{$\begin{array}{l}\text { CMOS } \\
\text { MEMS }\end{array}$} \\
\hline $\begin{array}{l}\text { Total chip size } \\
\left(\mathrm{mm}^{2}\right)\end{array}$ & \multicolumn{2}{|c|}{36} & \multicolumn{2}{|c|}{9} & \multicolumn{2}{|c|}{ N/A } & \multicolumn{2}{|c|}{2.46} \\
\hline Sensing type & \multicolumn{2}{|c|}{ Piezoresistive } & \multicolumn{2}{|c|}{ Capacitive } & \multicolumn{2}{|c|}{ Piezoresistive } & \multicolumn{2}{|c|}{ Capacitive } \\
\hline $\begin{array}{l}\text { Measurement } \\
\text { Range }(\mathrm{G})\end{array}$ & \multicolumn{2}{|c|}{ \pm 10} & \multicolumn{2}{|c|}{ \pm 1} & \multicolumn{2}{|c|}{ \pm 25} & \multicolumn{2}{|c|}{ $\pm 0.8 \sim 6$} \\
\hline \multirow{3}{*}{ Sensitivity $(\mathrm{mV} / \mathrm{G})$} & $\mathrm{X}$ & 31 & $\mathrm{X}$ & 520 & $\mathrm{X}$ & 0.22 & $\mathrm{X}$ & 0.53 \\
\hline & $\mathrm{Y}$ & 31 & $\mathrm{Y}$ & 460 & $\mathrm{Y}$ & 0.27 & $\mathrm{Y}$ & 0.28 \\
\hline & $\mathrm{Z}$ & 167 & Z & 320 & Z & 0.79 & $\mathrm{Z}$ & 0.2 \\
\hline Circuit gain $(\mathrm{dB})$ & \multicolumn{2}{|c|}{40} & \multicolumn{2}{|c|}{44.5} & \multicolumn{2}{|c|}{ N/A } & \multicolumn{2}{|c|}{0} \\
\hline $\begin{array}{c}\text { Cross-Axis } \\
\text { sensitivity (\%) }\end{array}$ & \multicolumn{2}{|c|}{$<7$} & \multicolumn{2}{|c|}{$<4.73$} & \multicolumn{2}{|c|}{$<5$} & \multicolumn{2}{|c|}{$<8.33$} \\
\hline
\end{tabular}

the X-, Y-, and Z-axis are $8.49 \mathrm{kHz}, 14.55 \mathrm{kHz}$, and $10.05 \mathrm{kHz}$, respectively, as summarized in Table I. The deviations between the measured and simulated results are $6.7 \%$ (X-axis), $7.8 \%$ (Y-axis), and 5.5\% (Z-axis), respectively. The deviations could be caused by the variations of structure dimensions from designed values after the fabrication processes. For instance, according to the TSMC user manual, the thickness variation of the metal/dielectric layers is $\pm 15 \%$. Such thickness variation will lead to a maximum deviation of natural frequencies for $13.4 \%$ (X-axis), $13.4 \%$ (Y-axis), and $13.3 \%$ (Z-axis), respectively. Moreover, the material properties, such as the elastic modulus and Poisson's ratio employed in the simulation model could differ from the real ones. The variation of material properties could also lead to a mismatch between the measured and predicted resonant frequencies. Compared with the design specifications predicted from the simulation in Table I, the measured noise floor in Table III is relatively high. The main reason for such a high noise floor is the initial displacement of the proof-mass caused by the predeformation of springs after the process. Thus, the initial sensing gap between the sensing electrodes deviates from the designed one. The initial displacement of sensing electrodes leads to unbalanced fully differential capacitance pairs and is detected by the readout circuit. Thus, the measured output noise shown in Table III consists of not only the Brownian noise and input referred circuit noise (as indicated in Table I) but also the noise from the initial position offset of the sensing electrodes. The initial position offset of the sensing electrodes even dominates the output noise. Moreover, as shown in Table I, the cross-axis sensitivity is ideally canceled by the fully differential sensing electrodes design. However, due to the initial displacement of sensing electrodes, higher cross-axis sensitivity will occur, as in the measurement results shown in Table III. In short, the initial displacements of the proof-mass and the sensing electrodes need to be considered to improve the performance of the tri-axis accelerometer. Finally, Table IV summarizes the comparison of the presented single proof-mass tri-axis accelerometer with other existing ones. Note that the circuit gain of [6] and [7] is more than $40 \mathrm{~dB}$, whereas the circuit of this paper is $0 \mathrm{~dB}$. Thus, 
the sensitivities of these two studies are much larger than that of the presented design.

\section{CONCLUSION}

This paper presents a novel design of single proof-mass tri-axis capacitive CMOS-MEMS accelerometer. Thus, the triaxis accelerometer and its capacitance readout IC are monolithically integrated on the same chip and the footprint of the chip is reduced by $50 \%$. Based on the CMOS process, the springs, especially the serpentine out-of-plane (Z-axis) spring, are designed to reduce the cross-axis sensitivity. Moreover, the in-plane and the out-of-plane sensing electrodes are also designed to suppress the cross-axis signal coupling. The tri-axis accelerometer has been successfully implemented using the standard TSMC 2P4M process and the in-house postprocessing. Measurement results show that the sensitivities (nonlinearity) of the accelerometer are, respectively: $0.53 \mathrm{mV} / \mathrm{G}(2.64 \%)$ in $\mathrm{X}$-axis, $0.28 \mathrm{mV} / \mathrm{G}(3.15 \%)$ in Y-axis, and $0.2 \mathrm{mV} / \mathrm{G}(3.36 \%)$ in $\mathrm{Z}$-axis. The cross-axis sensitivity ranges from $1 \%$ to $8.3 \%$ and the measurement range is between $0.8 \sim 6 \mathrm{G}$. In summary, the presented single proof-mass tri-axis CMOS-MEMS accelerometer offers reasonable sensitivities and acceptable cross-axis sensitivity.

\section{ACKNOWLEDGMENT}

The authors would like to thank TSMC Ltd., and National Chip Implementation Center (CIC), Taiwan, for supporting the IC Manufacturing. The authors would also like to thank National Tsing Hua University and National Chiao Tung University for providing the fabrication facilities.

\section{REFERENCES}

[1] M. Parameswaran, R. Chung, M. Gaitan, R. B. Johnson, and M. Syrzycki, "Commercial CMOS fabricated integrated dynamic thermal scene simulator," in IEDM Tech. Dig., Washington, DC, Dec. 8-11, 1991, pp. 754-756.

[2] K. H. L. Chau, S. R. Lewis, Y. Zhao, R. T. Howe, S. F. Bart, and R. G. Marcheselli, "An integrated force-balanced capacitive accelerometer for low g application," Sens. Actuators A, Phys., vol. 54, no. 1-3, pp. 472476, 1996.

[3] P. Dong, X. Li, H. Yang, H. Bao, W. Zhou, S. Li, and S. Feng, "Highperformance monolithic triaxial piezoresistive shock accelerometers," Sens. Actuators A, Phys., vol. 141, no. 2, pp. 339-346, Feb. 2008.

[4] J. Chae, H. Kulah, and K. Najafi, "A monolithic three-axis micro-g micromachined silicon capacitive accelerometer," J. Microelectromech. Syst., vol. 14, no. 2, pp. 235-241, Apr. 2005.

[5] M. A. Lemkin, M. A. Ortiz, N. Wongkomet, B. E. Boser, and J. H. Smith, "A 3-axis surface micromachined $\Sigma \Delta$ accelerometer," in Proc. IEEE 44th ISSCC, San Francisco, CA, Feb. 6-8, 1997, pp. 202-203.

[6] H. Takao, H. Fukumoto, and M. Ishida, "A CMOS integrated three-axis accelerometer fabricated with commercial submicrometer CMOS technology and bulk-micromachining," IEEE Trans. Electron Devices, vol. 48, no. 9, pp. 1961-1968, Sep. 2001.

[7] H. Qu, D. Fang, and H. Xie, "A monolithic CMOS-MEMS 3-axis accelerometer with a low-noise, low-power dual-chopper amplifier," IEEE Sensors J., vol. 8, no. 9, pp. 1511-1518, Sep. 2008.

[8] H. Luo, G. Zhang, L. R. Carley, and G. K. Fedder, "A post-CMOS micromachined lateral accelerometer," J. Microelectromech. Syst., vol. 11, no. 3, pp. 188-195, Jun. 2002.

[9] Analog Device Inc., "ADXL 335- Small, Low Power, 3-Axis $\pm 3 \mathrm{~g}$ Accelerometer," Norwood, MA, Jul. 2009, Rev. A.

[10] STMicroelectronics, "LIS331DLH: MEMS Digital Output Motion Sensor Ultra Low-Power High Performance 3-Axes Nano Accelerometer," Jul. 2009, Rev. 3.
[11] J. C. Lee, "Hacking the Nintendo Wii remote," Pervasive Comput., vol. 7, no. 3, pp. 39-45, Jul. 2008.

[12] M. A. Lemkin, B. E. Boser, D. Auslander, and J. H. Smith, "A 3-axis balanced accelerometer using a single proof-mass," in Proc. Transducer, Chicago, IL, Jun. 16-19, 1997, pp. 1185-1188.

[13] C.-M. Sun, C.-W. Wang, and W. Fang, "On the sensitivity improvement of CMOS capacitive accelerometer," Sens. Actuators A, Phys., vol. 141, no. 2, pp. 347-352, Feb. 2008.

[14] C. Wang, M.-H. Tsai, C.-M. Sun, and W. Fang, "A novel CMOS out-ofplane accelerometer with fully-differential gap-closing capacitance sensing electrodes," J. Micromech. Microeng., vol. 17, no. 7, pp. 1275-1280, Jun. 2007.

[15] O. Paul and H. Baltes, "Novel fully CMOS-compatible vacuum sensor," Sens. Actuators A, Phys., vol. 46/47, pp. 143-146, 1995.

[16] M.-H. Tsai, C.-M. Sun, C. Wang, J. Lu, and W. Fang, "A monolithic 3D fully-differential CMOS accelerometer," in Proc. IEEE NEMS, Sanya, China, Jan. 6-9, 2008, pp. 1067-1170.

[17] J. M. Tsai and G. K. Fedder, "Mechanical noise-limited CMOS-MEMS accelerometers," in Proc. IEEE MEMS, Miami, FL, Jan. 30-Feb. 2, 2005, pp. 630-633.

[18] Freescale Semicond., "Accelerometer Terminology Guide," Austin, TX, May 2007, Rev. 0.

[19] K. Kwon and S. Park, "A bulk-micromachined three-axis accelerometer using silicon direct bonding technology and polysilicon layer," Sens. Actuators A, Phys., vol. 66, no. 1-3, pp. 250-255, Apr. 1998.

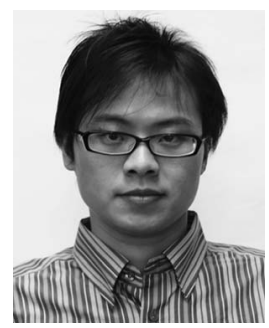

Chih-Ming Sun was born in Taipei, Taiwan, in 1981. He received his Master degree from the Institute of Nanoengineering and Microsystems, National Tsing Hua University, Hsinchu, Taiwan, in 2006. Currently, he is studying towards the Ph.D. degree in the Institute of Nanoengineering and Microsystems, National Tsing Hua University.

From April 2009 to January 2010, he was with Prof. G. K. Fedder at Carnegie Mellon University, Pittsburgh, PA, as a visiting student. His major research interests include CMOS-MEMS capacitive inertial sensors, CMOS-MEMS post processing, and sensors integration.

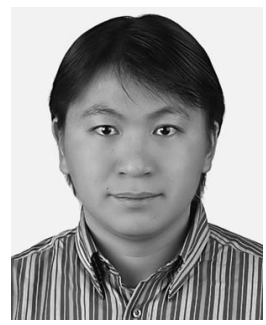

Ming-Han Tsai was born in Yunlin, Taiwan, in 1982. He received his Master degree from the Institute of Nanoengineering and Microsystems, National Tsing Hua University, Hsinchu, Taiwan, in 2007. Currently, he is studying towards the Ph.D. degree in the Institute of Nanoengineering and Microsystems, National Tsing Hua University.

His major research interests include CMOSMEMS sensors and actuators, and CMOS-MEMS postprocessing. 


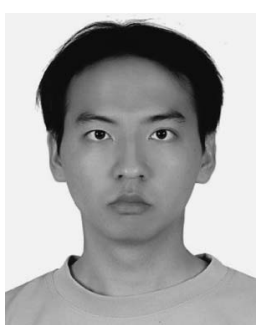

Yu-Chia Liu was born in Kaohsiung, Taiwan, in 1985. He received his Master degree from the Institute of Nanoengineering and Microsystems, National Tsing Hua University, Hsinchu, Taiwan, in 2009. Currently, he is studying towards the Ph.D. degree in the Institute of Nanoengineering and Microsystems, National Tsing Hua University.

His major research interests include CMOSMEMS capacitive sensing interface, CMOS-MEMS sensor, and readout circuit design.

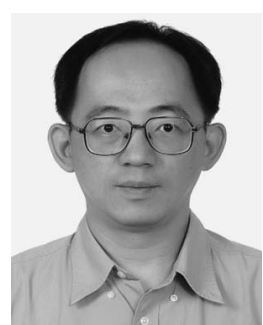

Weileun Fang (M'06) was born in Taipei, Taiwan. He received his Ph.D. degree from Carnegie Mellon University, Pittsburgh, PA, in 1995. His doctoral research focused on the determination of the mechanical properties of thin films using micromachined structures.

In 1995, he worked as a postdoctoral research at Synchrotron Radiation Research Center, Taiwan. He joined the Power Mechanical Engineering Department at the National Tsing Hua University, Hsinchu, Taiwan, in 1996, where he is now a Professor, as well as a faculty, of the NEMS Institute. In 1999, he was with Prof. Y.-C. Tai at the California Institute of Technology, Pasadena, California, as a visiting associate. His research interests include MEMS with emphasis on microfabrication/ packaging technologies, CMOS-MEMS, CNT-MEMS, microoptical systems, microsensors and actuators, and characterization of thin film mechanical properties.

Dr. Fang is now the Board Member of JMM and the Associate Editor of JM3. He has served as the chief delegate of Taiwan for the World Micromachine Summit, since 2008. He also served as the TPC of IEEE MEMS'04, MEMS'07, and MEMS' 10 , the regional TPC of Transducers' 07 , and the EPC of Transducers'09. He has become the member of the international steering committee of Transducers from 2009. Moreover, he also serves as a technical consultant for many MEMS companies in Taiwan. 